\title{
Paraganglioma del filum terminal como causa de síndrome de cauda equina
}

\section{Cauda equina syndrome caused by paraganglioma of the filum terminale}

\author{
J. Undabeitia-Huertas ${ }^{1}$ R. Noboa ${ }^{1}$, R. Jové ${ }^{2}$, M. Boix ${ }^{3}$, S. Gatius ${ }^{4}$ y P. Nogues ${ }^{1,5}$
}

\section{RESUMEN}

Los paragangliomas son tumores neuroendocrinos originados a partir de células que migran de la cresta neural. Su localización es diversa, siendo frecuentes en cabeza, cuello, mediastino o retroperitoneo. Su crecimiento en la región del filum terminal es muy poco frecuente. Presentamos el caso de una paciente que debuta con un cuadro agudo de cauda equina. Describimos en detalle el proceso diagnóstico, las características radiológicas, el tratamiento y las propiedades macro y microscópicas de este tumor.

\begin{abstract}
Paragangliomas are neuroendocrine tumors that originate from cells migrating from the neural crest. They have a diverse localizations, and are common in the head, neck, mediastinum or retroperitoneum. Their growth in the filum terminale region is very infrequent. We report the case of a patient who suffered an acute cauda equina syndrome. We give a detailed description of the diagnostic process, radiological characteristics, treatment and the macro and microscopic properties of this tumor.
\end{abstract}

1. Servicio de Neurocirugía. Hospital Universitario Arnau de Vilanova. Lleida

2. Servicio de Traumatología. Hospital Universitario Arnau de Vilanova. Lleida

3. Servicio de Neurología. Hospital Universitario Arnau de Vilanova. Lleida

4. Servicio de Anatomía Patológica. Hospital Universitario Arnau de Vilanova. Lleida

5. Departamento de Cirugía. Hospital Universitario Arnau de Vilanova. Lleida

\section{Autor responsable:}

José Undabeitia

Servicio de Neurocirugía

Hospital Universitario Arnau de Vilanova

Avenida Alcalde Rovira Roure

25198 Lleida

E-mail: joseundabeitia@hotmail.com

Los autores declaran no tener conflicto de intereses. 


\section{INTRODUCCIÓN}

Los paragangliomas son tumores endocrinos infrecuentes que pueden ubicarse en cualquier lugar del organismo y resultan de la migración de células de la cresta neural, en asociación al sistema nervioso autónomo ${ }^{1-8}$.

Estos tumores son más frecuentes en cabeza y cuello, mediastino, retroperitoneo y región para-aórtica. En relación al sistema nervioso central, su localización más frecuente son el cuerpo carotídeo, el glomus yugular y la cauda equina ${ }^{1-3,5,6,9-13}$.

Histológicamente los paragangliomas de la cauda equina son tumores benignos, que habitualmente se localizan el en filum terminal ${ }^{1,5}$.

\section{CASO CLÍNICO}

Presentamos el caso de una mujer de 47 años que acudió al servicio de Urgencias de nuestro hospital por un cuadro de dolor lumbar intenso de aparición brusca con dificultad para la marcha, que progresó rápidamente hacia un cuadro de paraplejia completa. Entre los antecedentes personales destacaban una alergia a la penicilina y una hipertensión arterial clasificada como idiopática, que fue diagnosticada siete años atrás y tratada con enalapril-hidroclorotiazida $(20-12.5 \mathrm{mg} / 24 \mathrm{~h})$. En la anamnesis la paciente refirió un dolor de inicio súbito, que le llegó a despertar por la noche. El dolor se irradiaba hacia ambas extremidades inferiores y dificultaba la marcha. La paciente negaba antecedente traumático alguno. En la exploración física inicial se objetivó un balance motor de $3 / 5$ en psoas y cuádriceps con $0 / 5$ en la flexo-extensión plantar. Igualmente se detectó una anestesia en la extremidad inferior derecha con hipoestesia termoalgésica en la extremidad inferior izquierda junto con anestesia perineal, globo vesical e incontinencia fecal. El balance motor en las extremidades superiores fue de $5 / 5$. La paciente no presentaba fiebre o signos de irritación meníngea. Se solicitó una tomografía axial computarizada (TAC) urgente de la región lumbar, que no mostró alteración alguna. Ante estos hallazgos se realizó una resonancia magnética (RMN) de columna lumbar (Fig. 1) que mostró la presencia de una lesión intradural que ocupaba la totalidad del saco dural en los niveles L2-L3-L4 con una lesión satélite asociada a nivel sacro.

Con la sospecha clínica y radiológica inicial de ependimoma se intervino de urgencia practicando una laminectomía y fijación pedicular L2-L3-L4. Al proceder a la apertura dural observamos una tumoración de color rojo violáceo, encapsulada, que desplazaba las raíces nerviosas

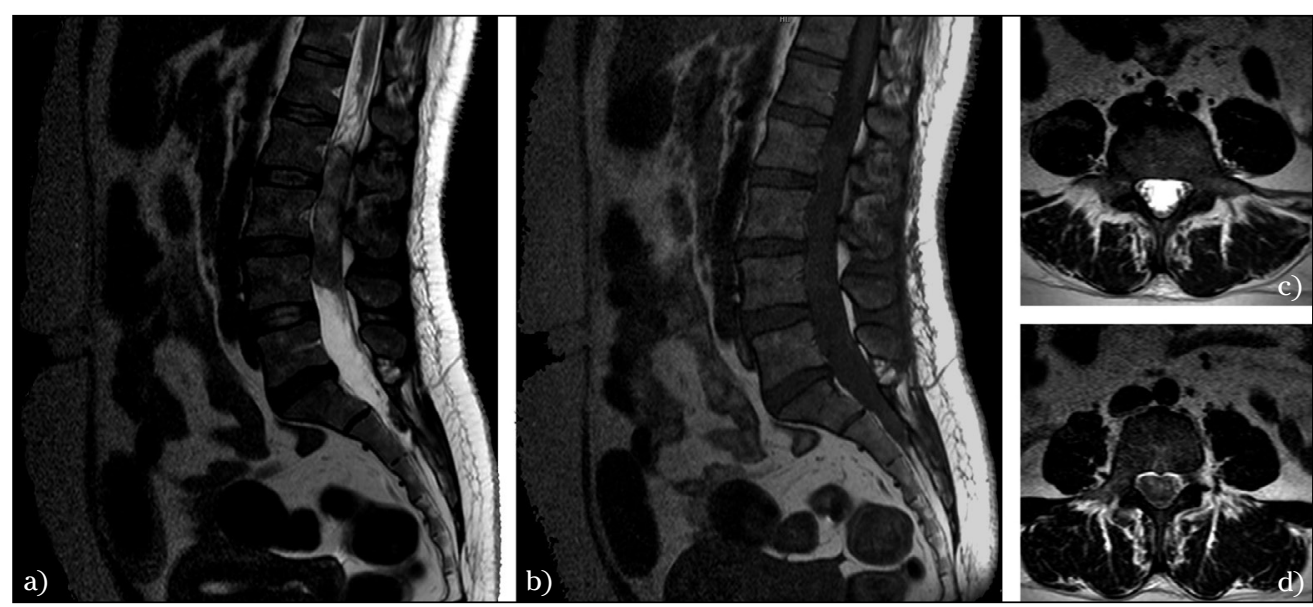

Figura 1. a) Corte sagital de RM de columna lumbar potenciada en T2 que muestra una lesión hiperintensa que ocupa en su totalidad el saco dural en los niveles L2-L3-L4 y una lesión satélite intradural a nivel de S2. b) Corte sagital de RM de columna lumbar potenciada en T1, en el que la misma lesión parece tener una intensidad similar al líquido cefalorraquídeo y al cono medular. $\mathrm{c}$ y d) Cortes axiales de RM de columna lumbar potenciada en T2 donde se aprecia el nivel L1 (c) -sin lesión-y el nivel inmediatamente inferior con una ocupación completa del canal (d). 

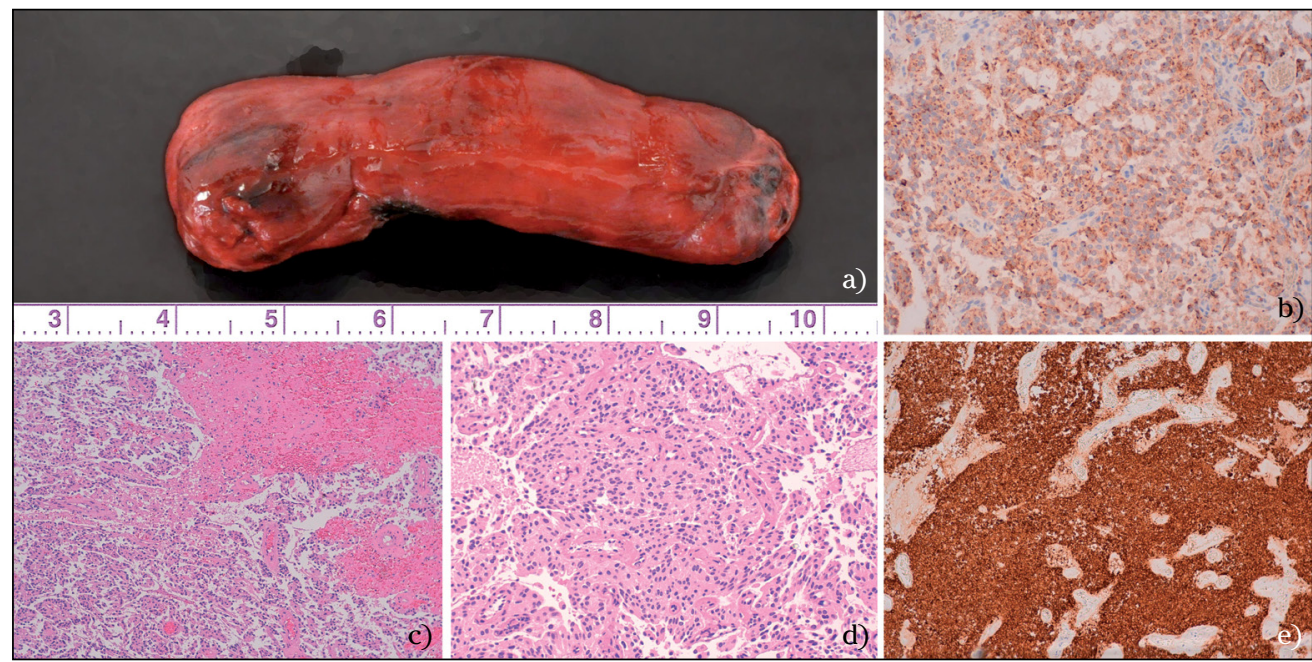

Figura 2. a) Aspecto macroscópico de la lesión, destacando una cápsula que delimita la lesión. b) H-E 10x. Proliferación celular trabecular y estructuras vasculares con focos de hemorragia. c) H-E 20x. Células sin atipia citológica con cromatina nuclear en patrón de "sal y pimienta". d) Positividad para cromogranina DAP 20x. e) Positividad para sinaptofisina 20x.

lateralmente. Mediante microcirugía se disecó con facilidad el tumor de las raíces adyacentes y se extirpó en su totalidad en una única pieza (fig. 2a). La lesión se encontraba adherida únicamente al filum terminal. El postoperatorio transcurrió sin complicaciones, experimentando una progresiva recuperación de la función motora e inicio de control de esfínteres. Ante el diagnóstico anatomopatológico de paraganglioma (fig. 2b-e), se solicitaron niveles de catecolaminas en sangre y en orina, resultando todos ellos normales. La paciente fue dada de alta a un centro rehabilitador para completar su recuperación.

\section{DISCUSIÓN}

Los paragangliomas de la región de la cauda equina son lesiones sólidas, ricamente vascularizadas, de crecimiento lento y bien encapsuladas, que habitualmente se originan a partir del filum terminal y sólo ocasionalmente en las raíces nerviosas ${ }^{1-5}$. El primer caso fue descrito por Millerty col en $1970^{14}$, que inicialmente lo describió como un ependimoma secretor ("secretory ependimoma"). Retrospectivamente se demostró que esta lesión se trataba de un paraganglioma de dicha localización ${ }^{1}$. Fue posteriormente, en 1972, cuando Lerman y $\mathrm{col}^{15}$ emplearon por primera vez el término "paraganglioma". Desde entonces se han descrito aproximadamente 220 casos en la literatura científica.

El origen de los paragangliomas de la cauda equina no se encuentra completamente aclarado, ya que la existencia de células paraganglionares en el sistema nervioso central continúa siendo objeto de debate. Algunos autores argumentan que las células paraganglionares se pueden hallar en la cauda equina, mientras que otros afirman que los paragangliomas en esta localización no son derivados de la cresta neural ${ }^{1,9,11}$.

Los paragangliomas del filum terminal son lesiones muy poco frecuentes $y$ representan entre un 2,5 y un $3,8 \%$ de los tumores en esta localización y pueden ser fácilmente confundidos por ependimomas o incluso schwannomas ${ }^{1,4,5,7,9}$. Su aparición se suele centrar en la etapa media de la vida, pero con un rango amplio de entre 13 a 71 años y cierta predilección por el sexo masculino $0^{9-11,13,16}$. 
Clínicamente, el síntoma inicial más frecuente es el dolor lumbar (50-87\% de los pacientes), que se puede acompañar de ciática (20-74\% de los casos) $)^{1,9-11}$. Su presentación de forma aguda es excepcional y normalmente se trata de un cuadro que varía entre meses o incluso años de evolución $^{3,10}$. La incidencia de trastornos motores o sensitivos, la disfunción de esfínteres o la hipertensión intracraneal son otros síntomas posibles, aunque estas formas de presentación son mucho menos habituales. La existencia de déficit motor o alteración de esfínteres se ha descrito como un factor de mal pronóstico funcional ${ }^{1,9,12}$.

El diagnóstico radiológico diferencial de estas lesiones resulta difícil ${ }^{4}$. La RMN es la prueba de elección para el estudio por imagen ${ }^{1,2}$. La lesión suele presentarse como hipo o iso intensa al cono medular en secuencias potenciadas en T1, mientras que se muestran hiperintensas en secuencias T2. Además de ello, captan intensamente gadolinio en las secuencias contrastadas debido a su importante vascularización ${ }^{1,2,5,10,13}$. En nuestro caso dicho contraste no fue administrado debido a que se trataba de un estudio urgente. Las características descritas no son específicas de este tipo de tumor y son comunes a otros tumores en esta localización ${ }^{2}$. Algunos autores han descrito la presencia de calcificaciones como un hallazgo sugestivo del paraganglioma, si bien, al igual que en nuestro caso, no siempre se hallan presentes. La TAC no tiene indicación alguna para el diagnóstico de estas lesiones en el momento actual, aunque algunos autores describen la presencia de signos indirectos, como el adelgazamiento de las láminas, debido a la presión ejercida por el tumor ${ }^{2,9}$.

En cuanto al diagnóstico endocrinológico los paragangliomas del filum terminal, a pesar de su origen neuroendocrino, raramente presentan actividad secretora ${ }^{1,6,10}$. En nuestro caso observamos un descenso mantenido de la tensión arterial, que inicialmente se interpretó como un cuadro de anemia debido a la reciente intervención, pero que ha permanecido constante en el tiempo y que ha permitido la retirada del tratamiento antihipertensivo previo. El estudio de catecolaminas fraccionadas en orina y en plasma, que solamente se realizó una vez conocido el diagnóstico anatomopatológico, reveló valores normales. No pudimos disponer de valores previos debido al inicio súbito del cuadro y a la urgencia del tratamiento quirúrgico. A diferencia de los paragangliomas en otras localizaciones, no se ha descrito en la literatura sintomatología relacionada con la producción de catecolaminas ${ }^{9}$.

El tratamiento consiste en la exéresis completa de la lesión, cuando ésta es posible $^{1,3-5,9,10,12}$. Los paragangliomas se clasifican como tumores de grado I según las World Health Organization (WHO), por lo que, cuando se consigue dicha exéresis radical, el tratamiento se considera curativo y la recurrencia puede suceder aquellos casos en los que solamente se puede conseguir una resección incompleta ${ }^{1,5,7,9}$. La aparición de una crisis catecolaminérgica durante la cirugía, tal como se ha descrito en los paragangliomas en otros niveles, no ha sido descrita en las lesiones de cauda equina $^{1,7}$. Cuando la cirugía programada es posible, la monitorización neurofisiológica intraoperatoria resulta de gran ayuda, especialmente en aquellos casos en los que la lesión se encuentra adherida a una raíz nerviosa ${ }^{4}$. En nuestro caso, dado que se trató de una cirugía urgente, esta monitorización no fue posible. Aunque algunos autores han sugerido la administración de tratamiento radioterápico tras una resección parcial, su utilidad en la prevención de la recurrencia en estos pacientes no ha sido demostrada ${ }^{1,4,16}$. Es necesario realizar un control a largo plazo de estos pacientes, debido al lento crecimiento de estas lesiones. Para dicho seguimiento, al igual que para su diagnóstico, la resonancia magnética es la prueba de elección. Tal como se ha presentado anteriormente, nuestra paciente presentaba una lesión satélite que no fue extirpada, por lo que este seguimiento es obligado $^{4,9,16}$.

Macroscópicamente, estos tumores presentan característicamente un aspecto rojo-violáceo, debido a su importante vascularización, bien circunscritos y de 
consistencia blanda (fig 2a) $)^{1,4,11,16}$. Histológicamente estos tumores son de naturaleza benigna y se caracterizan por la presencia de células con abundantes gránulos neurosecretores en su citoplasma ${ }^{1}$. Presentan una gran similitud con los ependimomas, que son los tumores más frecuentes en esta localización ${ }^{3}$. La característica histológica primordial es la presencia de grupos de células en "nidos" o "Zellballen" con cordones de células en el interior del estroma

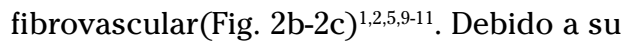
similitud con otros tumores es necesaria la realización de estudios de inmunohistoquimia $^{1,2,5,11}$, que característicamente va a mostrar la presencia de enolasa neuronal específica, proteína s 100, una cromogranina y sinaptofisina positivas (Figs. 2d-e) en la mayoría de los casos y una proteína gliofibrilar ácida negativa, lo que lo va a diferenciar de los ependimomas ${ }^{2,4}$

\section{BIBLIOGRAFÍA}

1. Ardon H, Plets C, Sciot R, Calenbergh FV. Paraganglioma of the cauda equina region: $\mathrm{A}$ report of three cases. Surg Neurol Int 2011; 2: 96.

2. Garcia-Maeso I, Figueredo-Mendez J, AlessandriniGonzalez R, Estupinan-Diaz B, Villegas-Anchon A, Piedra-Garcia $J$ et al. Cauda equina paraganglioma: a case report. Rev Neurol 2004; 39: 1197-1199.

3. ANDERSON JR, GulLAN RW. Paraganglioma of the cauda equina: a case report. J Neurol Neurosurg Psychiatry 1987; 50: 100-103.

4. Mathon B, Carpentier A, Clemenceau S, Boch AL, Bitar A, Mokhtari K et al. Paraganglioma of the cauda equina region: Report of six cases and review of the literature. Neurochirurgie 2012; 58: 341-345.

5. Midi A, Yener AN, SAv A, Cubuk R. Cauda equina paraganglioma with ependymoma-like histo- logy: a case report. Turk Neurosurg 2012; 22 : 353-359.

6. van Alphen HA, Bellot SM, Stam FC. Paraganglioma of cauda equina. Clin Neurol Neurosurg 1977; 79: 316-322.

7. Demircivi Ozer F, Aydin M, Bezircioglu H, Oran I. Paraganglioma of the cauda equina: a highly vascular tumour. J Clin Neurosci 2010; 17: 1445-1447.

8. Landi A, Tarantino R, Marotta N, Rocco P, AntoNELLI M, SALVATI M et al. Paraganglioma of the filum terminale: case report. World J Surg Oncol 2009; 7: 95.

9. Nagata K, Kakizoe M, TAKagi H, Ohashi T, Yamamoto S, Inoue A et al. Paraganglioma of the cauda equina. Kurume Med J 1988; 35: 95-99.

10. Walsh JC, O’Brien DF, Kumar R, Rawluk D. Paraganglioma of the cauda equina: a case report and literature review. Surgeon 2005; 3: 113116.

11. Singh RV, Yeh JS, BRoome JC. Paraganglioma of the cauda equina: a case report and review of the literature. Clin Neurol Neurosurg 1993; 95: 109-113.

12. Marcol W, Kiwic G, MalinowsKa-Kolodziej I, KotUlska K, Kotas A, AdAmeK D et al. Paraganglioma of the cauda equina presenting with erectile and sphincter dysfunction. J Chin Med Assoc 2009; 72: 328-331.

13. Faro SH, Turtz AR, Koenigsberg RA, Mohamed FB, Chen CY, Stein H. Paraganglioma of the cauda equina with associated intramedullary cyst: MR findings. AJNR Am J Neuroradiol 1997; 18: 1588-1590.

14. Miller CA, Torack RM. Secretory ependymoma of the filum terminale. Acta neuropathol 1970; 15: 240-250.

15. LeRman RI, Kaplan ES, Daman L. Ganglioneuroma-paraganglioma of the intradural filum terminale. Case report. J Neurosurg 1972; 36 : 652-658.

16. IliYa AR, Davis RP, SEIDMAn RJ. Paraganglioma of the cauda equina: case report with magnetic resonance imaging description. Surg Neurol 1991; 35: 366-367. 